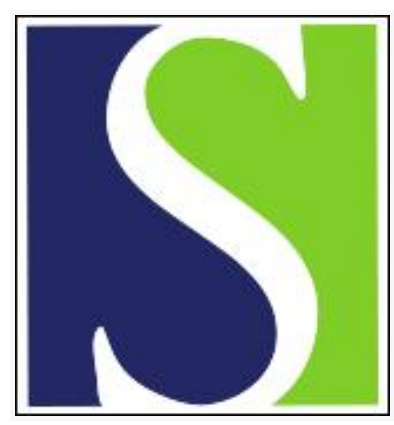

Scand J Work Environ Health 1987;13(4):326-329

https://doi.org/10.5271/sjweh.2033

Issue date: Aug 1987

Intraneural edema following exposure to vibration.

by Lundborg G, Dahlin LB, Danielsen N, Hansson HA, Necking LE, Pyykko I

Affiliation: Department of Hand Surgery, Malmo General Hospital, Sweden.

This article in PubMed: www.ncbi.nlm.nih.gov/pubmed/3433033

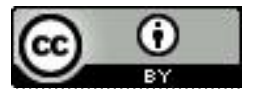




\title{
Intraneural edema following exposure to vibration
}

\author{
by Göran Lundborg, ${ }^{1,2}$ Lars B Dahlin, ${ }^{2}$ Nils Danielsen, ${ }^{2}$ Hans A Hansson, ${ }^{3}$ Lars E Necking, ${ }^{1}$ \\ IImari Pyykkö 4
}

\begin{abstract}
LUNDBORG G, DAHLIN LB, DANIELSEN N, HANSSON HA, NECKING LE, PYYKKÖ I. Intraneural edema following exposure to vibration. Scand J Work Environ Health 13 (1987) 326-329. Peripheral neuropathy represents a well-known complication from long-term exposure to vibration. In the present study an experimental model is presented with the purpose of analyzing the formation of intraneural edema following vibration exposure. Vibration $(82 \mathrm{~Hz}$, peak-to-peak amplitude $0.21 \mathrm{~mm}$ ) was induced in the hind limb of rats by the use of vibrating electric motors during $4 \mathrm{~h} / \mathrm{d}$ for $5 \mathrm{~d}$. Tracer techniques (with albumin Evans blue and horseradish peroxidase) were used to study the permeability of intraneural microvessels after the vibration exposure on day 5. It was found that the vibration trauma in this model induced epineurial edema in the sciatic nerve. It is hypothesized that the formation of intraneural edema may be an important pathophysiological factor in the occurrence of vibration-induced neuropathy.
\end{abstract}

Key terms: blood-nerve barrier, endoneurial fluid pressure, intraneural edema, intraneural microcirculation, nerve edema, peripheral neuropathy, rat, vibration injury.

\begin{abstract}
"White fingers" (Raynaud's phenomenon) and peripheral neuropathy represent well-known complications from long-term exposure to vibration. Although the peripheral vascular probiem has gained much attention $(1,2,17)$, the neurological disturbances have not been so well recognized. However, over recent years numbness, paresthesia, and muscle wasting have been observed, often in association with impaired cutaneous vibrotactile sensibility (5), decreased nerve conduction velocity (14), and decreased muscle power of the hand grip $(2,3,4)$.
\end{abstract}

In the upper extremity the neuropathy usually involves the median or ulnar nerves, more seldom both at one and the same time (7). In early stages the symptoms may imitate those seen in carpal tunnel syndrome although conduction velocity across the carpal tunnel is sometimes normal. The pathoanatomic basis for these lesions is not known, although some authors claim that they have observed degenerative fiber changes in nerves subjected to vibration (6) and in biopsies from fingers of vibration-exposed workers (16).

Peripheral nerves are well vascularized structures, responding to trauma with an inflammatory reaction including increased vascular permeability and the formation of intraneural edema $(8,9,10)$. An intraneural edema may produce serious acute consequences for normal nerve fiber function by changing the endoneu-

\footnotetext{
1 Department of Hand Surgery, Malmö General Hospital, Malmö, Sweden.

2 Laboratory of Experimental Biology, Department of Anatomy, University of Göteborg, Göteborg, Sweden.

3 Institute of Neurobiology, University of Göteborg, Göteborg, Sweden.

4 Institute of Occupational Health, Helsinki, Finland.
}

Reprint requests to: Dr G Lundborg, Department of Hand Surgery, Malmö General Hospital, S-214 01 Malmö, Sweden. rial composition of electrolytes and increasing endoneurial fluid pressure $(9,11,13)$. Chronic edema may be invaded by fibroblasts and transformed to an intraneural scar. There is reason to believe that the early stages of carpal tunnel syndrome, characterized by reversible nocturnal paresthesias in the hand, are based upon edema formation and vascular insufficiency occurring in the nerve during the night $(10,15)$.

The purpose of the present investigation was to study the effects of vibration on intraneural microvascular function with special reference to the formation of intraneural edema. Our hypothesis was that intraneural edema might be an etiologic factor in the occurrence of vibration-induced neuropathy.

\section{Materials and methods}

The study was carried out on Sprague Dawley rats (body weight $200-250 \mathrm{~g}$ ), anesthetized by intraperitoneal injections of a solution composed of sodium pentobarbital $(60 \mathrm{mg} / \mathrm{ml})$, $0.9 \%$ saline, and diazepam $(5 \mathrm{mg} / \mathrm{ml})$ in $1: 1: 4$ volume proportions. Vibration of controlled frequency and intensity was induced in one of the hind limbs of the rats during $4 \mathrm{~h} / \mathrm{d}$ for $5 \mathrm{~d}$. After the vibration exposure and perfusion by a tracer (see the following text) on the fifth day, the animals were killed, and the sciatic nerves were removed.

\section{The vibration exciter}

A simple method was developed for inducing vibration in the hind limb of the rats. Small electric motors were modified so that a counterweight of defined mass was fixed to the axis in an eccentric position. Increasing the voltage of the motor resulted in vibration of increasing frequency. At $14 \mathrm{~V}$ the motor induced vibration of $82 \mathrm{~Hz}$ and a peak-to-peak amplitude of $0.21 \mathrm{~mm}$ in the system.

The motor was enclosed in a silicone block. To compensate for the temperature increase in the system, air of room temperature was passed through two channels in the silicone mass close to the motor. A slit fitting the shape of the foot of the rats was made in the silicone block. The rats were sus- 
pended in a cradle with one hind foot placed in the slit. In this manner, the hind foot and limb were subjected to vibration of defined frequency, amplitude, and duration.

\section{Methods for assessing vascular permeability}

Evans blue-albumin. Serum albumin, labeled with Evans blue, has been used previously in numerous studies for assessing microvascular permeability in peripheral nerves $(8,9)$. A solution, here called EBA, was prepared by mixing $5 \%$ bovine albumin with Evans blue. After the vibration exposure on day 5 , EBA ( $1 \mathrm{ml}$ per $100 \mathrm{~g}$ of body weight) was slowly injected into a tail vein or via a catheter in the femoral vein of the limb not subjected to vibration and allowed to circulate for $30 \mathrm{~min}$. A segment of the sciatic nerve from the vibrated leg was then removed and fixed in $4 \%$ buffered formaldehyde solution for about $24 \mathrm{~h}$. Frozen longitudinal and transverse sections were mounted in $50 \%$ aqueous glycerine and examined in a Leitz fluorescence microscope. When studied in a fluorescence microscope, equipped with proper filter combinations $(8,12)$, EBA elicits a bright red-yellow fluorescence, while the nerve tissue elicits a green autofluorescence. Leakage of albumin through the walls of intraneural microvessels can hereby be easily detected.

Horseradish peroxidase. Horseradish peroxidase (MW 42.000) (HRP) has been used as a tracer in numerous investigations to study the permeability of intraneural microvessels and the perineurum. This substance can be detected by light microscopic, as well as ultrastructural, techniques. In the present study HRP ( $400 \mathrm{mg} / \mathrm{kg}$ of body weight) was slowly injected into a tail vein or via a catheter in the femoral vein of the contralateral leg and allowed to circulate for $30 \mathrm{~min}$. The vascular system of the rats was then rinsed by transcardial perfusion with physiological saline, and fixation was performed with $3 \%$ cacodylate-buffered glutaraldehyde. Segments of the sciatic nerve of the vibrated leg were then removed, fixed for $24 \mathrm{~h}$ in $3 \%$ glutaraldehyde, and transferred to a $7.5 \%$ sucrose solution. Sections were treated with diaminebenzodine and hydrogen peroxidate for light microscopic demonstration of the peroxidase. HRP was easily detected as a brown-red substance, in contrast to the white color of the nerve tissue.

\section{Results}

\section{Macroscopic appearance}

The nerve trunks of the vibrated leg were surrounded by a gelatinous edema (figure 1), which appeared generally spread in the subcutaneous space. The normal striated appearance of the superficial layer of the nerve had disappeared (figures 1 and 2), the nerve exhibiting an opaque homogeneous surface indicating epineurial edema.

\section{Evans blue-albumin}

In the vibrated leg of five rats, fluorescence microscopy revealed a diffuse red fluorescence confined to the epineurial tissue indicating protein leakage and epineurial edema (figure 3). In contrast, the nerve fibers inside the fascicles exhibited a normal green autofluorescence, and no red fluorescence (EBA) could be detected outside the vessels in the endoneurial space.

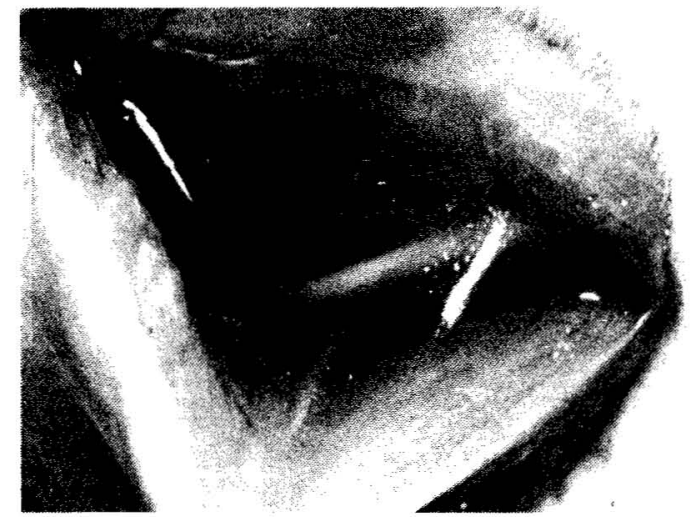

Figure 1. Gross appearance of a sciatic nerve and surrounding tissues in a leg subjected to vibration. Generalized subcutaneous edema. Diffuse, nonstriated surface of the nerve (arrow).

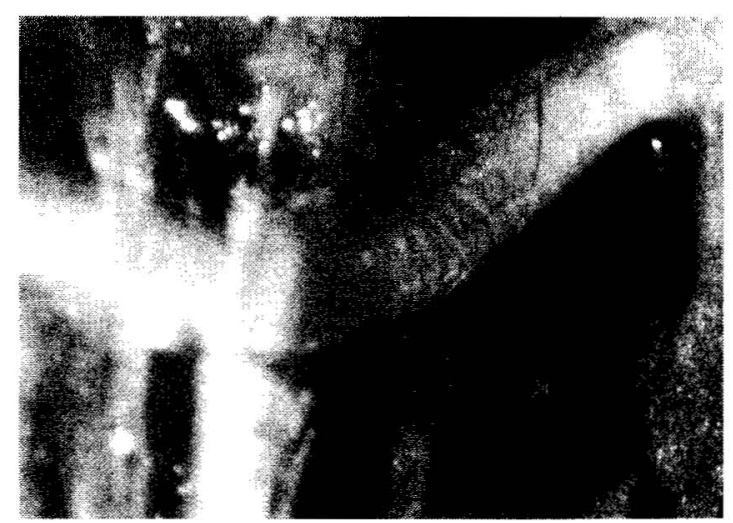

Figure 2. Appearance of the sciatic nerve surface of a control specimen at higher magnification. Note the striated superficial pattern, not recognized on corresponding nerve in the vibrated leg (figure 1).

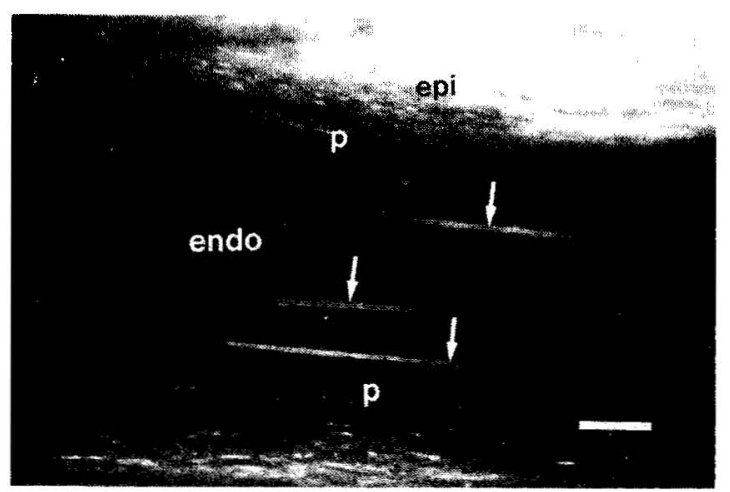

Figure 3. Longitudinal section of sciatic nerve. Epineurial edema in vibrated limb indicated by diffuse red (here white) fluorescence outside a fascicle. Inside the fascicles the fluorescence is confined to endoneurial capillaries (arrows). ( $p=$ perineurium, delimiting the fascicle, epi=epineurium, endo $=$ endoneurium, scale bar $=100$ microns) 


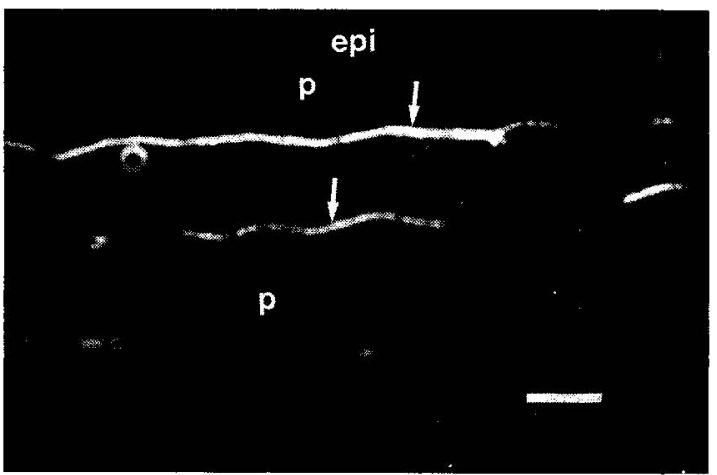

Figure 4. Control specimen. No red (here white) fluorescence in the epineurium. ( $p=$ perineurium, delimiting the fascicle, epi $=$ epineurium, scale bar $=100$ microns)

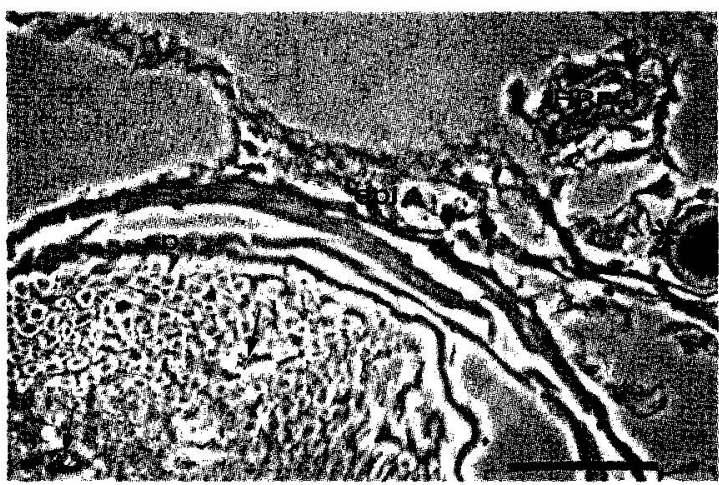

Figure 5. Transverse section of the sciatic nerve following vibration. Horseradish peroxidase (HRP), exhibiting a brown color, is confined to the lumen of the endoneurial capillaries (arrows) but is spread outside the epineurial vessels (*). ( $p=$ perineurium, scale bar $=100$ microns $)$

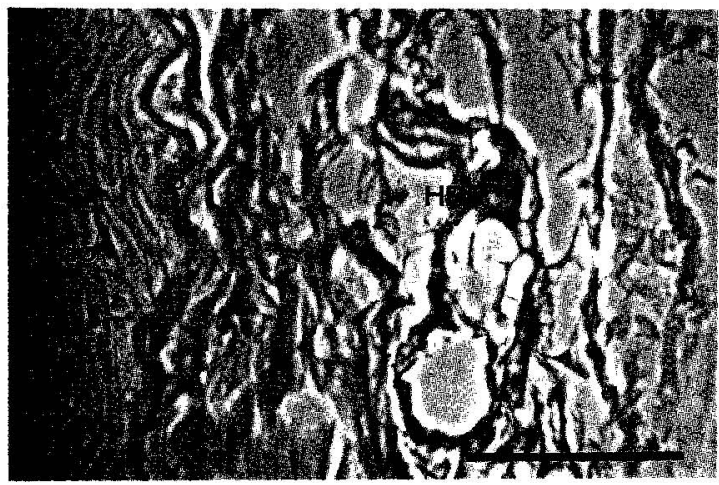

Figure 6. Diffuse horseradish peroxidase (HRP) spreading in epineurial tissue after vibration. $(\mathrm{p}=$ perineurium, endo $=$ endo neurium, scale bar $=100$ microns)
In the contralateral limbs (controls) neither the epineurium nor the endoneurium showed any signs of leakage of the dye complex, ie, the EBA was confined to the vessel lumina in both tissue layers (figure 4).

\section{Horseradish peroxidase}

In the light microscopic analysis in the case of the five rats which received HRP, HRP could be found confined to the lumen of the endoneurial capillaries. There was, however, a leakage of the substance through the walls of the epineurial vessels in nerves in the vibrated limb (figure 5), where HRP could be traced diffusely in the epineurial connective tissue (figure 6). The picture was consistent with slight epineurial edema. There were no detectable amounts of HRP in the epineurium of the sciatic nerve of the contralateral leg.

\section{Discussion}

The structure and function of the intraneural microvessels normally, and under certain pathological conditions, have been extensively outlined in numerous previous studies $(8,9)$. There are well developed vascular plexuses in all layers of the nerve. Anastomoses between these vascular plexuses are extensive, and the nerve has a significant "buffer capacity" to compensate for the effects of local vascular trauma. The endoneurial capillaries possess a "blood-nerve barrier" corresponding to the "blood-brain barrier" of the central nervous system. It prevents macromolecules in the blood from reaching the endoneurial space. This barrier, together with the diffusion barrier in the perineurium $(12,18)$, serves to maintain a specialized intrafascicular environment, optimizing nerve fiber function. The epineurial vessels do not possess such a diffusion barrier and react more easily to trauma by increasing their permeability. Epineurial edema is therefore seen at an early stage following ischemic, mechanical, and chemical trauma (8), while increased permeability of endoneurial vessels is seen first at a later stage in such instances.

In the present experimental model the vibration trauma induced epineurial edema. Such an edema might, in itself, interfere with nerve fiber nutrition by causing a "backward failure" in the nerve, including venular stasis and impaired oxygen supply to the nerve fibers. Hyperirritability of fibers, based upon hypoxia, results in paresthesias and numbness of the fingers. Clinically, the nocturnal, rapidly reversible paresthesias seen in carpal tunnel syndrome probably correspond to a nocturnal occurrence of such an intraneural edema, which, during the day is drained parallel to disappearance of the symptoms. If the edema becomes permanent, the symptoms also become permanent. A chronic epineurial edema may be invaded by fibroblasts and transformed into an intraneural constricting scar. In 
such instances the symptoms are persistent and may, ultimately, be associated with nerve fiber loss and muscle wasting.

Clinical experience shows that, when symptoms similar to those seen in patients with carpal tunnel syndrome occur in male patients, the condition is often associated with a long-term use of hand-held vibrating tools. However, several of these cases - in contrast to true carpal tunnel syndrome - do not present any nerve conduction abnormalities in the median nerve at the level of the carpal tunnel. We hypothesize that the symptoms in these instances are based upon intraneural edema in distal branches of the median nerve at the level of the finger pulps. The edema has been initiated by vibration exposure and aggravated during the night. The specialized anatomy of the finger pulps is characterized by numerous strong septum formations, constituting walls of tissue compartments. During the night the horizontal position of the body, as well as the absence of a muscle pump, contributes to an increase in tissue pressure in all tissue compartments of the upper extremity, including the compartments of the finger pulps. A nocturnal increase in tissue pressure in such compartments could be expected to affect terminal, edematous nerve branches and thereby elicit paresthesia within skin areas innervated of the median nerve. The clinical picture may imitate the carpal tunnel syndrome, but, if the median nerve is not involved at the more proximal carpal tunnel level, nerve conduction is normal across the wrist, and decompression by splitting the carpal ligament could not be expected to cure the condition.

\section{Acknowledgments}

This work was supported by grants from the Swedish Work Environmental Fund and the Swedish Medical Research Council (no 5188).

\section{References}

1. Agate JN. An outbreak of cases of Raynaud's disease of occupational origin. Br J Ind Med 6 (1949) 144-163.

2. Brammer AJ, Taylor W. Vibration effects on the hand and arm in industry. John Wiley \& Sons. New York, NY 1982.

3. Färkkilä M, Pyykkö I, Korhonen O, Starck J. Vibrationinduced decrease in the muscle force in lumberjacks. Eur J Appl Physiol 43 (1980) 1-8.

4. Färkkilä M, Starck J, Aatola S, Pyykkö J, Korhonen $O$. Vibration-induced neuropathy among forest workers. Acta Neurol Scand 71 (1985) 221-225.

5. Hagberg M, Johansson R, Zetterlund B. Tactile perception in workers with vibration syndrome. In: Institute of Occupational Health. Fourth international symposium on hand-arm vibration, Helsinki, May 6-8, 1985 . Helsinki 1985. (Abstract).

6. Karpova NI. Histopathology of the peripheral nerve stems subjected to the action of vibration. Russ Arch Pat 7 (1963) 49-53.

7. Lukáš E. Peripheral nervous system and hand-arm vibration exposure. In: Brammer AJ, Taylor W, ed. Vibration effects on the hand and arm in industry. John Wiley \& Sons, New York, NY 1982, pp 39-44.

8. Lundborg G. Ischemic nerve injury. Scand J Plast Reconstr Surg Suppl 6 (1970) 1-113.

9. Lundborg G. Structure and function of the intraneural microvessel as related to trauma, oedema formation and nerve function. J Bone Jt Surg 57-A (1975) 938-948.

10. Lundborg $G$. The intrinsic vascularization of human peripheral nerves: Structural and functional aspects. J Hand Surg 4 (1979) 34-41.

11. Lundborg G, Myers R, Powell H. Nerve compression injury and increase in endoneurial fluid pressure: $\mathrm{A}$ "miniature compartment syndrome." J Neurol Neurosurg Psychiatry 46 (1983) 1119-1124.

12. Lundborg G, Nordborg C, Rydevik B, Olsson Y. The effect of ischemia on the permeability of the perineurium to protein tracers in rabbit tibial nerve. Acta Neurol Scand 49 (1973) 287-294.

13. Myers R, Powell HC. Endoneurial fluid pressure in peripheral neuropathies. In: Hargens AR, ed. Tissue fluid pressure and composition. Williams \& Wilkins, Baltimore, London 1981, pp 193-207.

14. Seppäläinen AM. Nerve conduction in the vibration syndrome. Work Environ Health 7 (1970) 82-84.

15. Sunderland $S$. The nerve lesion in the carpal tunnel syndrome. J Neurol Neurosurg Psychiatry 39 (1976) 615626.

16. Takeuchi T, Futatsuka M, Imanishi H, Yamada S. Pathological changes observed in the finger biopsy of patients with vibration-induced white finger. Scand J Work Environ Health 12 (1986) 280—283.

17. Taylor W. The vibration syndrome. Academic Press, London, New York 1974.

18. Thomas PK, Olsson Y. Microscopic anatomy and function of the connective tissue components of peripheral nerve. In: Dyck PJ, Thomas PK, Lambert EH, Bunge R, ed. Peripheral neuropathy. Volume 1. WB Saunders, Philadelphia, PA 1984, pp 97-120. 